\title{
Chemical Evolution of Protostars
}

\author{
Michiel R. Hogerheijde \\ Leiden Observatory, P.O. Box 9513, 2300 RA, Leiden, The Netherlands
}

\begin{abstract}
This review discusses the evolution of the chemical composition of the molecular gas throughout the process of star formation. It covers the initial stages of cloud-core formation, gravitational collapse and the formation of the star, the effects of stellar radiation and outflows on the surrounding cloud, and the composition of the circumstellar disk. A brief overview of relevant chemical processes sets the stage. The ensuing evolution of the chemistry is governed by several competing timescales and by energy input by the young star(s). Special attention is given to the role of molecular freeze-out on dust grains and to deuterium fractionation in cold gas.
\end{abstract}

Keywords: Star Formation, Astrochemistry, Molecular Astrophysics

\section{Introduction}

Molecular lines offer powerful probes of interstellar clouds and starforming regions. Because excited energy levels are populated through collisions, relative line strengths of transitions of the same molecule provide measures of the gas density and temperature. The (emission or absorption) line profiles directly reflect the velocity field along the line of sight. And changes in the chemical abundances can signal specific processes such as shocks, heating, and photodissociation.

Full exploitation of these diagnostics requires understanding of the chemical processes that govern the molecular abundances. Ultimately, this may allow use of the chemistry as a clock or at least as a tool to assign a relative evolutionary ordering of objects. The following sections describe the basic chemical processes and sketch the evolution of the chemistry throughout star formation; more detailed reviews include van Dishoeck \& Blake (1998), van Dishoeck \& Hogerheijde (1999), and Langer et al. (2000).

\section{Chemical Processes}

At the low densities of interstellar clouds, chemical reactions in the gas phase involve only two bodies. Three-body reactions only play a role in shocks and the inner regions of protoplanetary disks, where $\mathrm{H}_{2}$ densities exceed $\sim 10^{12} \mathrm{~cm}^{-3}$. For two body reactions, the reaction rates (in $\mathrm{cm}^{-3} \mathrm{~s}^{-1}$ ) can be expressed as $k n(X) n(Y)$, where $k$ is 
Table I. Formation and Destruction Reactions

\begin{tabular}{ll}
\hline Radiative association & $\mathrm{X}+\mathrm{Y} \leftrightarrow \mathrm{XY}^{*} \leftrightarrow \mathrm{XY}+\nu$ \\
Photodissociation & $\mathrm{XY}+\nu \rightarrow \mathrm{X}+\mathrm{Y}$ \\
Associative detachment & $\left\{\begin{array}{l}\mathrm{X}+\mathrm{e} \rightarrow \mathrm{X}^{-}+\nu \\
\mathrm{X}^{-}+\mathrm{Y} \rightarrow \mathrm{XY}+e\end{array}\right.$ \\
Dissociatve recombination & $\mathrm{X}^{+}+\mathrm{e} \rightarrow \mathrm{X}+\nu$ \\
& $\mathrm{XY}+\mathrm{e} \rightarrow\left\{\begin{array}{l}\mathrm{XY}+\nu \\
\mathrm{X}+\mathrm{Y}\end{array}\right.$ \\
Gas-phase neutral-neutral reaction & $\mathrm{X}+\mathrm{YZ} \rightarrow \mathrm{XY}+\mathrm{Z}$ \\
Gas-phase ion-neutral reaction & $\mathrm{X}^{+}+\mathrm{YZ} \rightarrow \mathrm{XY}+\mathrm{Z}$ \\
Charge transfer & $\mathrm{X}^{+}+\mathrm{YZ} \rightarrow \mathrm{X}+\mathrm{YZ}^{+}$ \\
Grain-surface reactions & $\mathrm{X}+\mathcal{G}: \mathrm{Y} \rightarrow \mathrm{X}: \mathcal{G}: \mathrm{Y} \rightarrow \mathcal{G}: \mathrm{XY} \rightarrow \mathcal{G}+\mathrm{XY}$ \\
\hline
\end{tabular}

the rate coefficient in $\mathrm{cm}^{3} \mathrm{~s}^{-1}$. Table I lists the main reactions (Dalgarno, 1987; van Dishoeck, 1988). On-line databases list the rates of all known reactions, e.g., the UMIST 'rate99' database which contains 4113 reactions between 396 species and 12 elements (Le Teuff et al., 2000).

Molecular bonds can either form through radiative association, where a photon carries off the excess energy, or on a grain surface, where the dust particle absorbs the released energy. Typical rates range from $<10^{-17}$ to $10^{-13} \mathrm{~cm}^{3} \mathrm{~s}^{-1}$ with uncertainties as large as a factor 10 . Ultraviolet radiation can easily break molecular bonds. In the unattenuated interstellar radiation field (Draine, 1978), molecular lifetimes are as short as $10^{2}-10^{3} \mathrm{yr}$. Deep inside clouds $\left(\mathrm{A}_{\mathrm{V}}>5 \mathrm{mag}\right)$, electrons released when cosmic-rays ionize $\mathrm{H}_{2}$ can collisionally excite other $\mathrm{H}_{2}$ molecules, which decay by emitting an ultraviolet photon. This secondary ultraviolet field photodissociates molecules at a rate $4-5$ orders of magnitude lower than on the outside of clouds.

Existing molecular bonds can be rearranged through gas-phase reactions between neutral species or between an ion and a neutral species. The latter reactions are fast, with rate coefficients of $10^{-7}-10^{-9} \mathrm{~cm}^{3} \mathrm{~s}^{-1}$; the former reactions are generally slower by several orders of magnitude. Many ion-molecule reaction pathways are started by the formation of $\mathrm{H}_{3}^{+}$initiated by cosmic-ray ionization of $\mathrm{H}_{2}$,

$$
\begin{aligned}
& \mathrm{H}_{2}+\mathcal{C R} \rightarrow \mathrm{H}_{2}^{+}+\mathrm{e}^{-} \\
& \mathrm{H}_{2}^{+}+\mathrm{H}_{2} \rightarrow \mathrm{H}_{3}^{+}+\mathrm{H} .
\end{aligned}
$$

Cosmic rays penetrate deep into dark clouds, providing a ubiquitous source of $\mathrm{H}_{3}^{+} . \mathrm{H}_{3}^{+}$reacts radipdly with, e.g., $\mathrm{CO}$ or $\mathrm{N}_{2}$, producing $\mathrm{HCO}^{+}$ and $\mathrm{N}_{2} \mathrm{H}^{+}$, and allowing further ion-molecule reactions. 
Reactions that do not proceed effectively in the gas-phase can occur on grain surfaces, after the reagents have accreted onto a grain and diffused along the surface to the reaction site. The timescale for a gas molecule to stick on a grain is

$$
t_{\mathrm{accr}} \approx 2 \times 10^{9}\left(n_{\mathrm{H}} y_{\mathrm{s}}\right)^{-1},
$$

where $y_{\mathrm{s}}=0.1-1.0$ is the probability that the molecule sticks (see Williams, 1993 for a review). If the temperature of the dust is below the value at which the ice species evaporates $(\sim 20 \mathrm{~K}$ for $\mathrm{CO}$, $\sim 90 \mathrm{~K}$ for $\mathrm{H}_{2} \mathrm{O}$ ), it will remain on the grain until desorbed by processes such as cosmic-ray spot heating, grain-grain collisions, and exo-thermic chemical reactions (e.g., Schutte, 1997). Many reactions can proceed on grain surfaces because of the large time available ( $\sim 1$ day), even when reaction barriers are present. Because $\mathrm{H}$ is the most mobile species, surface chemistry leads to hydrogenated species such as $\mathrm{CH}_{4}, \mathrm{H}_{2} \mathrm{O}$, and $\mathrm{NH}_{3}$. Grain surfaces are the primary site for the formation of $\mathrm{H}_{2}$.

An extensive literature exists on modeling the chemistry of interstellar clouds. The interested reader is referred to the reviews listed in $\S 1$. Generally, one can distinguish models including only gas-phase reactions and those including also freeze-out, grain-surface reactions, and desorption. One can also distinguish time-dependent models, which follow the evolution over time, and depth-dependent models, which are calculated until chemical equilibrium is reached $\left(10^{5}-10^{7} \mathrm{yr}\right)$. The dynamical timescales involved in star formation are comparable in magnitude, requiring that the chemistry is solved both as function of time and position. Current computational capabilities do not permit to do this efficiently for the thousands of reactions of the full networks. Ruffle et al. (2002), Wiebe et al. (2003), and Semenov et al. (2004) have developed reduced networks that make such calculations tractable.

\section{Evolution of the Chemistry}

\subsection{Diffuse Clouds}

Diffuse clouds have extinction up to a few magnitudes and $\mathrm{H}+\mathrm{H}_{2}$ densities less than a few thousand $\mathrm{cm}^{-3}$. These clouds are poorly shielded from the interstellar ultraviolet radiation field, which plays a significant role in their chemsitry. In general, the abundances in these clouds are well explained by simple ion-neutral gas-phase chemistry; exceptions are the abundances of $\mathrm{NH}_{3}, \mathrm{H}_{2} \mathrm{CO}$, and $\mathrm{H}_{2} \mathrm{~S}$, which may be formed on grains and require a desorption mechanism. Meyer (1997) and Lucas \& Liszt (1997) review observational aspects of diffuse-cloud chemistry. 


\subsection{Dark Clouds}

Dark clouds have extinctions $>$ few mag, and shield their molecules from the dissociating ultraviolet interstellar radiation field. $\mathrm{H}_{2}$ and ${ }^{12} \mathrm{CO}$ exist in sufficient abundance that they can shield themselves against photodissociation; other species are shielded by dust. The densities of dark clouds are higher than those of diffuse clouds $\left(\gtrsim 10^{4} \mathrm{~cm}^{-3}\right)$ and their temperatures lower (as low as $10 \mathrm{~K}$ ). More complex species can survive here. Unsaturated carbon chains form through carbon insertion (e.g., $\mathrm{C}^{+}+\mathrm{CH}_{4} \rightarrow \mathrm{C}_{2} \mathrm{H}_{2}^{+}+\mathrm{H}_{2}$ or $\mathrm{C}+\mathrm{C}_{2} \mathrm{H}_{2} \rightarrow \mathrm{C}_{3} \mathrm{H}+\mathrm{H}$ ), condensation (e.g., $\mathrm{CH}_{3}^{+}+\mathrm{CH}_{4} \rightarrow \mathrm{C}_{2} \mathrm{H}_{5}^{+}+\mathrm{H}_{2}$ or $\mathrm{C}_{2} \mathrm{H}+\mathrm{C}_{2} \mathrm{H}_{2} \rightarrow \mathrm{C}_{4} \mathrm{H}_{2}+\mathrm{H}$ ), or radiative association $\left(\mathrm{C}^{+}+\mathrm{C}_{\mathrm{n}} \rightarrow \mathrm{C}_{\mathrm{n}+1}^{+}+\nu\right)$.

A classic example of a dark cloud is TMC 1, in the Taurus-Auriga cloud complex. This filamentary cloud shows prominent gradients in abundance along its length in species such as $\mathrm{NH}_{3}$ and $\mathrm{HC}_{7} \mathrm{~N}$ (Olano et al., 1988). Because of the similarity of dynamical and chemical timescales, explanations for this gradient invoke either a density slope along the ridge or a difference in evolutionary stage. The latter may be the result from turbulence affecting one side of the cloud and effectively resetting the chemical clock.

This dynamic interpretation of molecular clouds has been recently proposed by Hartmann et al. (2001), based on the distribution of premain-sequence stellar ages in Solar-neighborhood clouds. Bergin et al. (2004) has shown that convergent flows can indeed form $\mathrm{H}_{2}$ and $\mathrm{CO}$ quickly once a sufficient column density has been accumulated for shielding to become effective. This underlines the relevance of timedependent chemical models.

\subsection{Depletion}

Equation (3) shows that as the density of a cloud increases, molecules freeze out more rapidly. Inside pre-stellar cores, $\mathrm{H}_{2}$ densities exceed $10^{5} \mathrm{~cm}^{-3}$, and $t_{\mathrm{accr}}<t_{\mathrm{dyn}}$. The pre-stellar core L1498 (Kuiper et al., 1996; Willacy et al., 1998) offers a clear example of molecular depletion through freeeze out. $\mathrm{C}^{18} \mathrm{O}, \mathrm{CS}$, and CCS emission form a ring around the core's center as traced by thermal continuum emission from dust.

Not all species drop in abundance. Volatile molecules such as $\mathrm{H}_{2}$ and $\mathrm{N}_{2}$ do not stick on the grains. Following reactions (1-2), $\mathrm{H}_{3}^{+}$can react with $\mathrm{N}_{2}$ to form $\mathrm{N}_{2} \mathrm{H}^{+}$and $\mathrm{NH}_{3}$. The main destruction reactions of $\mathrm{N}_{2} \mathrm{H}^{+}$are with $\mathrm{CO}$ and $\mathrm{H}_{2} \mathrm{O}$, both of which are depleted inside cloud cores, and $\mathrm{N}_{2} \mathrm{H}^{+}$can retain significant abundances. The prestellar cores L1498 and B68 (Bergin et al., 2002) show $\mathrm{NH}_{3}$ and $\mathrm{N}_{2} \mathrm{H}^{+}$ emission peaks at their center. At the very center of $\mathrm{B} 68$, however, $\mathrm{N}_{2} \mathrm{H}^{+}$too decreases, suggesting that even $\mathrm{N}_{2}$ ultimately depletes.

hogerheijde.tex; 20/05/2004; $16: 08 ;$ p.4 

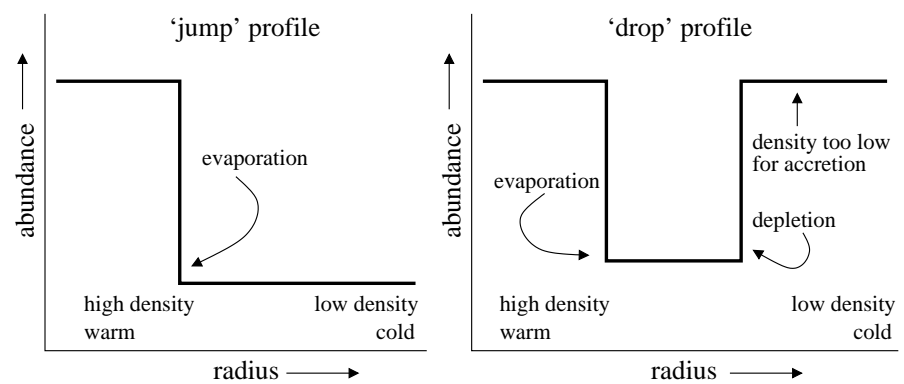

Figure 1. 'Jump' (left) and 'drop' (right) abundance profiles, after Van der Tak et al. (2000) and Jørgensen et al. (2004).

Studying the velocity fields in pre-stellar cores is critical to understand the earliest phases of collapse. The depletion of so many molecules poses a challenge to our ability to trace these earliest stages of collapse. Only $\mathrm{H}_{3}^{+}$and its isotopes (§3.4) may offer observational access.

\subsection{Deuteration}

The cosmic abundance of deuterium is $[\mathrm{D}] /[\mathrm{H}] \approx 1.6 \times 10^{-5}$, but deuterated forms of many molecules are observed at much higher relative abundance. Deuterium fractionation starts with the exchange reaction

$$
\mathrm{H}_{3}^{+}+\mathrm{HD} \leftrightarrow \mathrm{H}_{2} \mathrm{D}^{+}+\mathrm{H}_{2} .
$$

Below $\sim 20 \mathrm{~K}$ the forward reaction is favored because of the lower zero-point vibrational energy of $\mathrm{H}_{2} \mathrm{D}^{+}$. This drives much of the atomic $\mathrm{D}$ into $\mathrm{H}_{2} \mathrm{D}^{+}$or even $\mathrm{HD}_{2}^{+}$, inserting $\mathrm{D}$ into the ion-neutral chemical network. In regions of high depletion the main destruction partner of $\mathrm{H}_{3}^{+}$(and isotopes), CO, is underabundant, increasing the likelihood of $\mathrm{H}_{2} \mathrm{D}^{+}$reacting with other species and further boosting the deuterium fractionation of the products. Detections of singly, doubly, and even triply deuterated forms of, e.g., $\mathrm{H}_{3}^{+}, \mathrm{H}_{2} \mathrm{CO}, \mathrm{NH}_{3}$, and $\mathrm{CH}_{3} \mathrm{OH}$ imply deuterium fractionation by up to four (!) orders of magnitude (Lis et al., 2002; van der Tak et al., 2002; Bacmann et al., 2003; Caselli et al., 2003; Ceccarelli et al., 2004; Parise et al., 2004).

\subsection{Jump AND Drop Profiles}

Once a star has formed at the center of the collapsing core, it will start to heat the infalling material. In ice mixtures, species can segregate and evaporate at their respective $T_{\text {evap }}$, or the inclusions can evaporate together with the $\mathrm{CO}$ or $\mathrm{H}_{2} \mathrm{O}$ matrix.

The resulting abundance distributions follow so-called 'jump' or ‘drop' profile (Fig. 3.5; van der Tak et al., 2000; Jørgensen et al., 2004). 


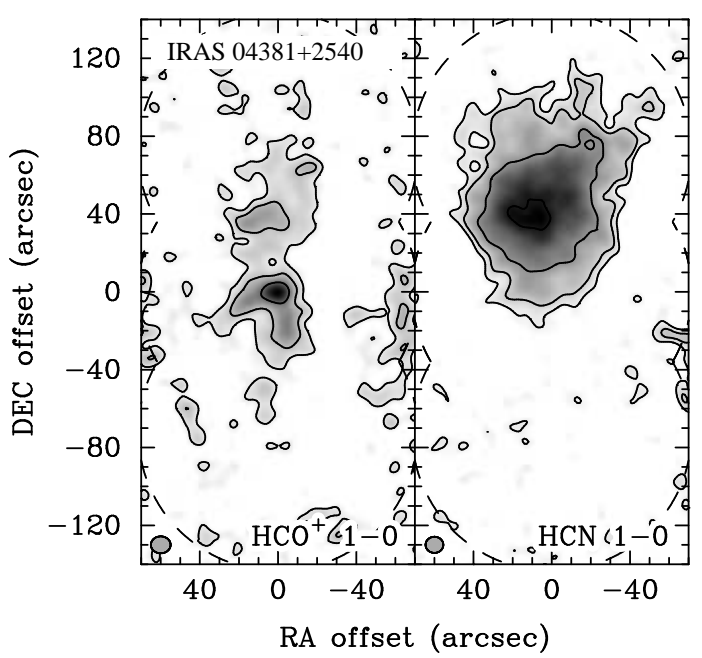

Figure 2. $\mathrm{HCO}^{+}$(left) and $\mathrm{HCN} J=1-0$ (right) emission image of the outflow driven by the young stellar object IRAS $04381+2540$ [at $(0,0)$ ] obtained with the BIMA interferometer by Hogerheijde (2001). The emission, especially of HCN in the northern outflow lobe, is strongly enhanced, suggesting significant abundance increases.

Species that form in the ice mantles on dust grains have 'jump' profiles, where the abundance is negligible for radii outside the location where $T_{\mathrm{d}}(r)=T_{\text {evap }}$, and high inside this radius. Species that were present in the gas-phase in the original cloud (e.g., CO), have a depleted ('drop') abundance for radii $r_{\text {evap }}<r<r_{\text {accr }}$, where $r_{\text {evap }}$ is the radius within which $T_{\mathrm{d}}(r)>T_{\text {evap }}$, and $r_{\text {accr }}$ the radius where $t_{\text {accr }}<t_{\text {age }}$. Outside $r_{\text {accr }}$ the density is too low to accrete significant amounts of the molecule within the life time of the core (eq. 3 ).

\subsection{OutFlows}

The bipolar jets that many young stars drive also affect the abundances in the surrounding cloud material by heating and shocking the gas, reviewed by Bachiller (1996). In shocks with speeds of $v_{s} \approx 25 \mathrm{~km} \mathrm{~s}^{-1}$ grains are destroyed or sputtered, and large columns for $\mathrm{SiO}$ rapidly form from the released Si (Schilke et al., 1997).

Weaker shocks at the sides of the flow can still evaporate the ice mantles of grains, releasing species such as $\mathrm{CH}_{3} \mathrm{OH}$ and $\mathrm{HCN}$ (Fig. 3.6; for another recent example see, e.g., Jørgensen et al., 2004). Large quantities of $\mathrm{H}_{2} \mathrm{O}$ are also released or formed (Bergin et al., 1998). As a result, species such as $\mathrm{N}_{2} \mathrm{H}^{+}$that are abundant in the unpertubed cloud are destroyed through reactions with water and absent from the shocked regions (Jørgensen et al., 2004). 


\subsection{Massive Star Formation}

The formation of massive stars is less well understood than that of lower mass objects. Massive stars are less common and often located at larger distances; much of their early evolution occurs deep in dense clouds; and massive stars often form together with many lower mass objects, complicating the interpretation of (unresolved) observations. Evans (1999) gives a comparison of low vs. high-mass star formation.

Nevertheless, the chemical evolution of sites of massive star formation is understood qualitatively. While governed by the same processes as lower mass objects, the much larger luminosity of massive stars greatly affects the outcome. The earliest observable state is that of a 'hot core', where the star has started to heat a sizeable fraction of its surrounding gas, evaporating many ice species. This is followed by an ultra-compact H II region (UCHII), where the ultraviolet radiation of the star has begun to photo-ionize part of its surroundings. In time, this will develop into a H II region. Space limitations preclude more details here; the interested reader is referred to the reviews of Kurtz et al. (2000) and Churchwell (2002).

\subsection{Protoplanetary Disks}

Many young stars are surrounded by circumstellar disks (Beckwith \& Sargent, 1996). The chemistry in these disks follows the same processes as described above: In the disk midplane, where densities are high and temperatures low, molecules are frozen out. At larger heights in the flared disk, molecules can exist in the gas-phase. At the surface of the flared disk, the ultraviolet radiation from the star dissociates and ionizes the material. Although low mass stars emit little ultraviolet radiation, mostly from accretion, the disk material is sufficiently close to the star that the ultraviolet radiation field at $100 \mathrm{AU}$ still equals 300-3000 times the interstellar field (Bergin et al., 2003).

Observations of molecular lines from disks have confirmed the picture sketched above: large depletions are inferred, and many molecules typically trace the warm gas at intermediate heights (e.g., Dutrey et al., 1997; van Zadelhoff et al., 2001). Signatures of photoprocessing such as the CN radical are present at large heights (Qi, 2001).

Current observations only have a few resolving elements across the disk, and no in-depth comparisons to theory can be made (yet). Open questions are the extent to which material mixes radially and vertically, which would expose a larger fraction of the disk material to different temperatures and radiation regimes. At the current relatively poor resolution, observations only trace material $>50 \mathrm{AU}$ which may never make it into a planetary system. 


\section{Summary and Outlook}

The chemistry in star-forming regions is governed by four competing processes: gas-phase chemistry, depletion (and grain-surface chemistry), evaporation, and photodissociation.

The differences in luminosity and ionizing flux between low mass and high mass objects causes large variations in the chemistry of the surrounding material, although the basic processes are the same.

The chemistry in disks also follows the same processes, but densities are higher, and even weak ionizing fluxes from the central star are important because of the small distances.

Much of what we know about this chemistry has been learned with single-dish telescopes like CSO and JCMT; and interferometer arrays like PdBI, OVRO, and BIMA. In the coming years, our understanding will greatly benefit from observations with APEX, the SMA and its link-up with JCMT and CSO, the merger of OVRO and BIMA into CARMA, and ultimately ALMA. These instruments will be complemented at shorter wavelengths by the Spitzer, Herchel, and SOFIA.

Together these observational possibilities will allow acces to higher excitations, tracing warmer and denser material; and higher spatial resolutions, allowing to separate the different regions that are still confused around high-mass stars and in protplanetary disks. Paired with increased modeling capabilities, the foreseen observations will greatly increase our understanding of the chemical evolution of protostars.

\section{References}

Bachiller, R.: 1996, 'Bipolar Molecular Outflows from Young Stars and Protostars', Annual Review of Astronomy and Astrophysics Vol. no. 34, pp. 111-154

Bacmann, A., Lefloch, B., Ceccarelli, C., Steinacker, J., Castets, A., and Loinard, L.: 2003, 'CO Depletion and Deuterium Fractionation in Prestellar Cores', Astrophysical Journal Vol. no. 585, pp. L55-L58

Beckwith, S. V. W. and Sargent, A. I.: 1996, 'Circumstellar disks and the search for neighbouring planetary systems.', Nature Vol. no. 383, pp. 139-144

Bergin, E. A., Neufeld, D. A., and Melnick, G. J.: 1998, 'The Postshock Chemical Lifetimes of Outflow Tracers and a Possible New Mechanism to Produce Water Ice Mantles', Astrophysical Journal Vol. no. 499, pp. 777-792

Bergin, E. A., Alves, J., Huard, T., and Lada, C. J.: 2002, ' $\mathrm{N}_{2} \mathrm{H}^{+}$and $\mathrm{C}^{18} \mathrm{O}$ Depletion in a Cold Dark Cloud', Astrophysical Journal Vol. no. 570, pp. L101-L104

Bergin, E., Calvet, N., D'Alessio, P., and Herczeg, G. J.: 2003, 'The Effects of UV Continuum and Ly $\alpha$ Radiation on the Chemical Equilibrium of T Tauri Disks', Astrophysical Journal Vol. no. 591, pp. L159-L162

Bergin, E. A., Hartmann, L. W., Raymond, J. C., and Ballesteros-Paredes, J.: 2004, 'Molecular Cloud Formation behind Shock Waves', Astrophysical Journal, in press (astro-ph/0405329) 
Caselli, P., van der Tak, F. F. S., Ceccarelli, C., and Bacmann, A.: 2003, 'Abundant $\mathrm{H}_{2} \mathrm{D}^{+}$in the pre-stellar core L1544', Astronomy and Astrophysics Vol. no. 403, pp. L37-L41

Ceccarelli, C., Dominik, C., Lefloch, B., Caselli, P., and Caux, E.: 2004, 'Detection of $\mathrm{H}_{2} \mathrm{D}^{+}$: Measuring the Midplane Degree of Ionization in the Disks of DM Tauri and TW Hydrae', Astrophysical Journal Vol. no. 607, pp. L51-L54

Churchwell, E.: 2002, 'Ultra-Compact HII Regions and Massive Star Formation', Annual Review of Astronomy and Astrophysics Vol. no. 40, pp. 27-62

Dalgarno, A.: 1987, 'Chemical processes in the interstellar gas', In NATO ASIC Proc. 210: Physical Processes in Interstellar Clouds, eds. G. Morfill, M. S. Schiler, Reidel, Dordrecht, pp. 219-239

Draine, B. T.: 1978, 'Photoelectric heating of interstellar gas', Astrophysical Journal Supplement Series Vol. no. 36, pp. 595-619

Dutrey, A., Guilloteau, S., and Guelin, M.: 1997, 'Chemistry of protosolar-like nebulae: The molecular content of the DM Tau and GG Tau disks.', Astronomy and Astrophysics Vol. no. 317, pp. L55-L58

Evans, N. J.: 1999, 'Physical Conditions in Regions of Star Formation', Annual Review of Astronomy and Astrophysics Vol. no. 37, pp. 311-362

Hartmann, L., Ballesteros-Paredes, J., and Bergin, E. A.: 2001, 'Rapid Formation of Molecular Clouds and Stars in the Solar Neighborhood', Astrophysical Journal Vol. no. 562, pp. 852-868

Jørgensen, J. K., Hogerheijde, M. R., Blake, G. A., van Dishoeck, E. F., Mundy, L. G., and Schöier, F. L.: 2004, 'The impact of shocks on the chemistry of molecular clouds. High resolution images of chemical differentiation along the NGC 1333-IRAS 2A outflow', Astronomy and Astrophysics Vol. no. 415, pp. 1021-1037

Jørgensen, J. K., Schöier, F. L., and van Dishoeck, E. F.: 2004, 'Molecular inventories and chemical evolution of low-mass protostellar envelopes', Astronomy and Astrophysics Vol. no. 416, pp. 603-622

Kuiper, T. B. H., Langer, W. D., and Velusamy, T.: 1996, 'Evolutionary Status of the Pre-protostellar Core L1498', Astrophysical Journal Vol. no. 468, pp. 761-773

Kurtz, S., Cesaroni, R., Churchwell, E., Hofner, P., and Walmsley, C. M.: 2000, 'Hot Molecular Cores and the Earliest Phases of High-Mass Star Formation', Protostars and Planets IV, eds. V. Mannings, A. P. Boss, S. S. Russell, Tucson, University of Arizona Press, pp. 299-326

Langer, W. D., van Dishoeck, E. F., Bergin, E. A., Blake, G. A., Tielens, A. G. G. M., Velusamy, T., and Whittet, D. C. B.: 2000, 'Chemical Evolution of Protostellar Matter', Protostars and Planets IV, eds. V. Mannings, A. P. Boss, S. S. Russell, Tucson, University of Arizona Press, pp. 29-57

Le Teuff, Y. H., Millar, T. J., and Markwick, A. J.: 2000, 'The UMIST database for astrochemistry 1999', Astronomy and Astrophysics Supplement Series Vol. no. 146, pp. 157-168

Lis, D. C., Roueff, E., Gerin, M., Phillips, T. G., Coudert, L. H., van der Tak, F. F. S., and Schilke, P.: 2002, 'Detection of Triply Deuterated Ammonia in the Barnard 1 Cloud', Astrophysical Journal Vol. no. 571, pp. L55-L58

Lucas, R., and Liszt, H. S.: 1997, 'Millimeter-wave Observations of Diffuse Clouds', In Proc. IAU Symp. 178, Molecules in Astrophysics: Probes and Processes, eds. E. F. van Dishoeck, Kluwer Academic Publishers, Dordrecht, pp. 421-430

Meyer, D. M.: 1997, 'Optical and Ultraviolet Observations of Diffuse Interstellar Clouds', In Proc. IAU Symp. 178, Molecules in Astrophysics: Probes and 
Processes, eds. E. F. van Dishoeck, Kluwer Academic Publishers, Dordrecht, pp. $407-419$

Olano, C. A., Walmsley, C. M., and Wilson, T. L.: 1988, 'The relative distribution of $\mathrm{NH}_{3}, \mathrm{HC}_{7} \mathrm{~N}$ and $\mathrm{C}_{4} \mathrm{H}$ in the Taurus Molecular Cloud 1 (TMC 1)', Astronomy and Astrophysics Vol. no. 196, pp. 194-200

Parise, B., Castets, A., Herbst, E., Caux, E., Ceccarelli, C., Mukhopadhyay, I., and Tielens, A. G. G. M.: 2004, 'First detection of triply-deuterated methanol', Astronomy and Astrophysics Vol. no. 416, pp. 159-163

Qi, C.: 2001, 'Aperture synthesis studies of the chemical composition of protoplanetary disks and comets', Ph.D. Thesis, California Institute of Technology

Ruffle, D. P., Rae, J. G. L., Pilling, M. J., Hartquist, T. W., and Herbst, E.: 2002, 'A network for interstellar CO - The first application of objective reduction techniques in astrochemistry', Astronomy and Astrophysics Vol. no. 381, pp. L13-L16

Schilke, P., Walmsley, C. M., Pineau des Forets, G., and Flower, D. R.: 1997, 'SiO production in interstellar shocks.', Astronomy and Astrophysics Vol. no. 321, pp. 293-304

Schutte, W. A.: 1997, 'Formation and evolution of interstellar icy grain mantles', NATO ASIC Proc. 48\%: The Cosmic Dust Connection, eds. J. M. Greenberg, Kluwer Academic Publishers, Dordrecht, pp. 177-201

Semenov, D., Wiebe, D., and Henning, T.: 2004, 'Reduction of chemical networks. II. Analysis of the fractional ionisation in protoplanetary discs', Astronomy and Astrophysics Vol. no. 417, pp. 93-106

van der Tak, F. F. S., van Dishoeck, E. F., and Caselli, P.: 2000, 'Abundance profiles of $\mathrm{CH}_{3} \mathrm{OH}$ and $\mathrm{H}_{2} \mathrm{CO}$ toward massive young stars as tests of gas-grain chemical models', Astronomy and Astrophysics Vol. no. 361, pp. 327-339

van der Tak, F. F. S., Schilke, P., Müller, H. S. P., Lis, D. C., Phillips, T. G., Gerin, M., and Roueff, E.: 2002, 'Triply deuterated ammonia in NGC 1333', Astronomy and Astrophysics Vol. no. 388, pp. L53-L56

van Dishoeck, E. F.: 1988, 'Molecular cloud chemistry', In Millimetre and Submillimetre Astronomy, eds. R. D. Wolstencroft, W. B. Burton, Kluwer Academic Publishers, Dordrecht, pp. 117-162

van Dishoeck, E. F. and Blake, G. A.: 1998, 'Chemical Evolution of Star-Forming Regions', Annual Review of Astronomy and Astrophysics Vol. no. 36, pp. 317368

van Dishoeck, E. F. and Hogerheijde, M. R.: 1999, 'Models and Observations of the Chemistry Near Young Stellar Objects', In NATO ASIC Proc. 540: The Origin of Stars and Planetary Systems, eds. C. J. Lada, N. D. Kylafis, Kluwer Academic Publishers, Dordrecht, pp. 97-140

van Zadelhoff, G.-J., van Dishoeck, E. F., Thi, W.-F., and Blake, G. A.: 2001, 'Submillimeter lines from circumstellar disks around pre-main sequence stars', Astronomy and Astrophysics Vol. no. 377, pp. 566-580

Wiebe, D., Semenov, D., and Henning, T.: 2003, 'Reduction of chemical networks. I. The case of molecular clouds', Astronomy and Astrophysics Vol. no. 399, pp. $197-210$

Willacy, K., Langer, W. D., and Velusamy, T.: 1998, 'Dust Emission and Molecular Depletion in L1498', Astrophysical Journal Vol. no. 507, pp. L171-L175

Williams, D. A.: 1993, 'Physical and Chemical Processes on Dust', Dust and Chemistry in Astronomy, eds. T. J. Miller, D. A. Williams, Institute of Physics Publishing, Philadelphia, p. 143 